\title{
Bitter Melon as a Therapy for Diabetes, Inflammation, and Cancer: a Panacea?
}

\author{
Deep Kwatra $^{1,2} \cdot$ Prasad Dandawate $^{1} \cdot$ Subhash Padhye $^{3} \cdot$ Shrikant Anant $^{1,2}$
}

Published online: 21 January 2016

(C) Springer International Publishing AG 2016

\begin{abstract}
Natural products have been used for centuries for cures prevention, treatment, and cure of multiple diseases. Some dietary agents are present in multiple systems of medicines as proposed treatments for chronic and difficult to treat diseases. Once such natural product is Momordica charantia or bitter melon. Bitter melon is cultivated in multiple regions across the world, and various parts of the plant, such as fruit, leaves seed, etc. have been shown to possess medicinal properties in ancient literature. Over the last few decades, multiple well-structured scientific studies have been performed to study the effects of bitter melon in various diseases. Some of the properties for which bitter melon has been studied include: antioxidant, antidiabetic, anticancer, anti-inflammatory, antibacterial, antifungal, antiviral, anti-HIV, anthelmintic, hypotensive, anti-obesity, immuno-modulatory, antihyperlipidemic, hepato-protective, and neuro-protective activities. This review attempts to summarize the various literature findings regarding medicinal properties of bitter melon. With such strong scientific support on so many medicinal claims, bitter melon comes close to being considered a panacea.
\end{abstract}

This article is part of the Topical Collection on Cancer Chemoprevention

Shrikant Anant

sanant@kumc.edu

1 Department of Surgery, University of Kansas Medical Center, 3901 Rainbow Boulevard, Kansas City, KS 66160, USA

2 The University of Kansas Cancer Center, University of Kansas Medical Center, 3901 Rainbow Boulevard, Kansas City, KS 66160, USA

3 Interdisciplinary Science and Technology Research Academy, Abeda Inamdar College, University of Pune, Pune 411001, India
Keywords Herbal remedy · Anticancer · Antidiabetic · Anti-inflammatory $\cdot$ Drug resistance $\cdot$ Dietary agent $\cdot$ Obesity

\section{Introduction}

Nature is full of deadly poisons as well as life-saving entities. In natural products such as medicinal plants, their extracts have been used for thousands of years in traditional medicine throughout the world. In the past century, new drug development is mainly being carried out using combinatorial chemistry and targeted drug design. However, phytochemicals and their analogs have also started to garner a lot of attention in drug discovery because of their relatively better safety profile, possible multi-targeted activities, and potential to treat wide range of diseases. A report from the World Health Organization (WHO) had reported that about $80 \%$ of the world's population utilizes traditional system of medicine as their first line of therapy [1]. Multiple countries in Asia, Africa, and the Middle East have long-standing and still prevailing systems of traditional medicines, most containing within them large compilations of therapeutic plants and related products [2]. Some of the medicinal plants described in Ayurveda (the traditional Indian system of medicine), viz. bitter melon (BM) [3-5], turmeric [6, 7], and stone apple [8, 9], have demonstrated their importance in recent years for treatment of various diseases including diabetes and cancer. With advancement in science and technology, it is now a lot more feasible to discover the active constituents present in these plants and establish their pharmacology for developing them into safer and more efficient therapeutics.

The current review discusses and summarizes the potential biological activities of BM or Momordica charantia (MC). BM, though a medicinal plant in Ayurveda and Chinese systems of medicine, is often consumed as a cooked vegetable in 
both these countries as well as other parts of Southeast Asia. It is known as bitter melon, bitter gourd, balsam pear, bitter cucumber, and Karela [10]. All parts of the plant, including the fruit, taste bitter. The shoot and leaves of bitter melon are used as vegetables for cooking, while fruit extracts are also utilized in tea preparations $[11,12]$. Since the bitterness of the vegetable is considered desirable, hence, the bitter flavor has been preferentially selected during domestication [13].

In Ayurveda, MC is claimed to possess antidiabetic, abortifacient, anthelmintic, antimalarial, and laxative properties. Additional indications include dysmenorrhea, emmenagogue, eczema, gout, galactagogue, kidney (stone), jaundice, leucorrhea, leprosy, pneumonia, piles, rheumatism, and psoriasis [14]. In recent years, MC and its various extracts have been studied for biological activities including antioxidant [15], antidiabetic [16], anticancer [3, 4], antiinflammatory [17], antibacterial [18], antifungal [19], antiviral [20], anti-HIV [21], anthelmintic [22], antimycobacterial [23], hypotensive [24], anti-obesity [25], Immuno-modulatory [26], antihyperlipidemic [27], hepatoprotective [28], and neuro-protective activities [29]. Numerous chemical constituents such as curcubitane-type triterpenoids, curcubitane-type glycosides, triterpene saponins, phenolic and flavonoid compounds, and protein components [30] have been identified and studied for various therapeutic activities. In the present review, we are summarizing some of the important reports dealing with antioxidant, anti-inflammatory, anticancer, and antidiabetic activities of MC. With so many health and therapeutic benefits, one can consider bitter melon to be as close to panacea as possible that exists in nature.

\section{M. charantia}

The genus Momordica occurs to subtribe Thladianthinae, tribe Joliffieae, subfamily Cucurbitoideae, of the Cucurbitaceae family [31]. The genus Momordica includes 45 plant species domesticated in Asia and Africa [32]. The plant grows in tropical areas of Asia, Amazon, east Africa, and the Caribbean. The core of MC domestication is mainly in eastern Asia, with greater proportion in India and southern China [33]. Other countries of prominent cultivation include Brazil, Colombia, Cuba, Ghana, Haiti, Mexico, Malaya, New Zealand, Nicaragua, Panama, and Peru [34, 35]. MC has been mentioned in Ayurvedic books from 2000 to $200 \mathrm{BCE}$ [32], providing reference of early cultivation of MC in India. The Chinese written reference to MC was documented in 1370 [33]. Both the domesticated and putative wild progenitors of $\mathrm{MC}$ are recorded in floras of India, tropical Africa, and Asia as well as Brazil, where it first came and then spread into Central America [13].

\section{Morphological Characteristics}

MC is an herbaceous plant with tendril-bearing vines that grows up to $5 \mathrm{~m}$ in length. It has simple and alternate leaves, which are $4-12 \mathrm{~cm}$ across. These leaves bear 3-7 deeply separated lobes. The plant has two different kinds of flowers separated based on sex. MC has easily differentiable male and female, yellowcolored flowers, owing to its monecious qualities. The male flowers grow at the end of thin, long stems, whereas the female flowers have a small fruit at the base of flower stem. The fruit is oblong in shape with a distinct warty on exterior. The cross-sections of the fruit show hollow interior with thin layer of flesh covering a central seed cavity filled with large, flat seeds as well as pith. Often, fruit is eaten when it is green or just starts turning yellow in color. At this stage, the fruit is crunchy as well as watery in texture. As the fruit progress to ripening, the rind becomes tough and with sometimes increased bitterness, while pith becomes sweet in taste and red in color. After full ripening, fruit turns orange in color, mushy, and often splits into segments, that curl back to expose seeds covered in bright red pulp (Fig. 1).

MC is available commercially in a variety of shapes and sizes. The Chinese variety is $20-30 \mathrm{~cm}$ long, oblong with bluntly tapering ends, and pale green colored with a gently undulating, warty surface, whereas the Indian variety has a narrower shape with pointed ends and a surface covered with jagged, triangular "teeth" and ridges. Indian $\mathrm{MC}$ is available in two varieties based on size, shape, color, and surface texture of the fruit. The first variety is $M$. charantia var. charantia having large fusiform of fruits that are not tapered at both ends and possesses numerous triangular tubercles looking like a "crocodile's back." The second variety is M. charantia var. muricato (Wild), which produces small and round fruits with tubercles, and more or less tapering at both ends [36].

\section{Nutritional Values}

The nutritional analysis of MC fruits shows that it is a rich in carbohydrates, proteins, vitamins, fibers, and minerals (Table 1). MC holds the highest nutritive value among all cucurbits [37]. Vitamin C content of Chinese MC differs significantly (440-780 $\mathrm{mg} / \mathrm{kg}$ edible portion). Considerable variation in nutrients, including protein, carbohydrates, iron, zinc, calcium, magnesium, phosphorous, and ascorbic acid, has been observed in MC [37, 38]. The pulp around the seeds of the mature ripe fruit is a major source of the carotenoid lycopene. 
Fig. 1 Raw and ripened fruit of bitter melon
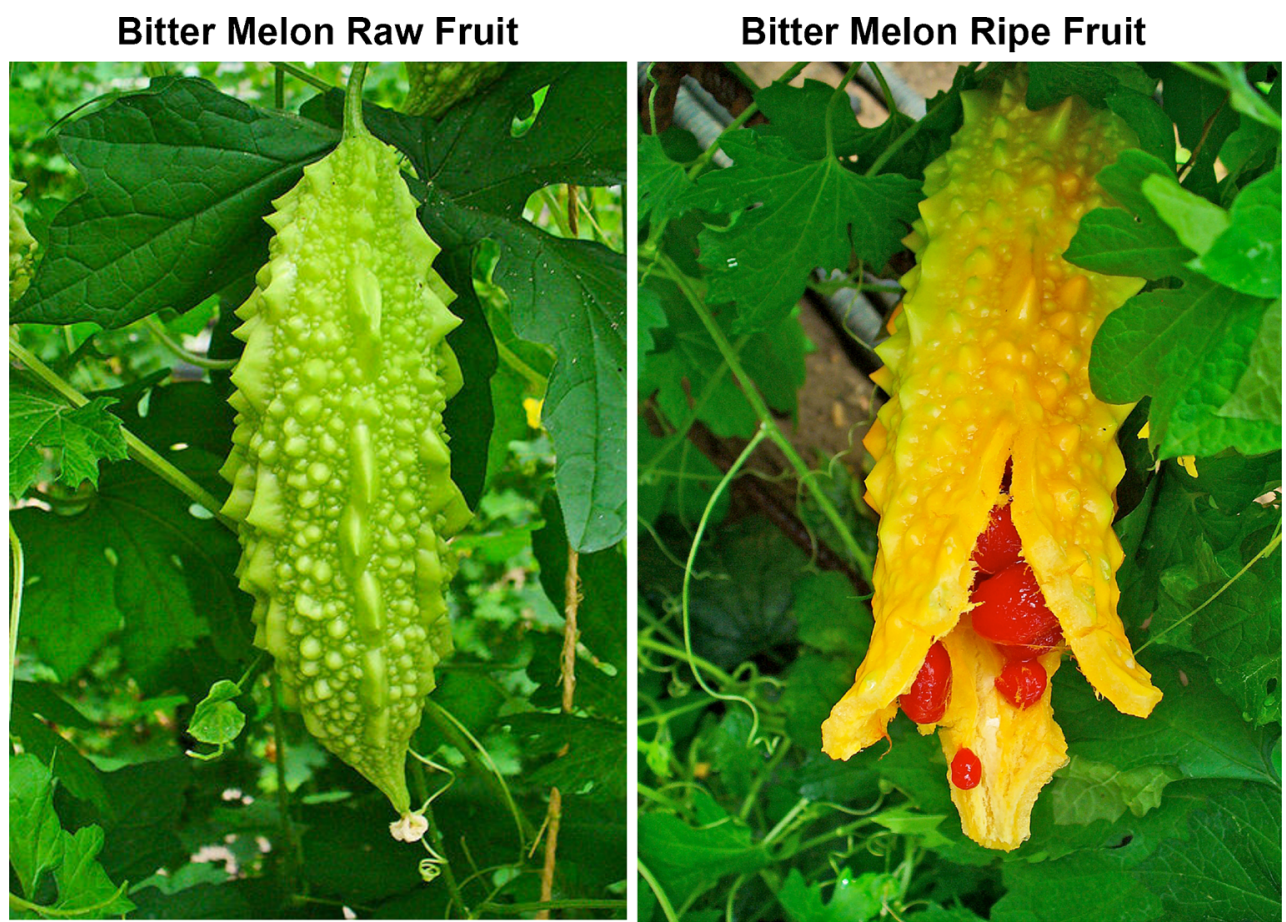

\section{Biological Activities of Bitter Melon}

\section{Antioxidant Activity and Antidiabetic Activity}

Oxidant production as normal metabolic by-products or their exposure from environment often leads to effects such as aging or other degenerative disorders. Consumption of antioxidant-rich foods can help alleviate such issues. Multiple reports using different extracts of BM have shown potential antioxidant properties of the fruit. $\mathrm{Lu}$ and group have studied the protective effects of the ethanolic extracts of MC fruit, against chronic alcohol-induced hepatic injury in C57BL/6 mice. It is believed that the protective effects are facilitated by improving levels of liver antioxidant enzymes (GSH, GPx, GRd, CAT, and SOD), decreasing lipid peroxidation (MDA), and lowering expression of pro-inflammatory cytokines (TNF- $\alpha$, IL-1 $\beta$, and IL-6) [28]. Similarly, antioxidant potential of MC extract was studied in ammonium chloride-induced (AC) hyperammonemic rats. Treatment with MC extract normalized the abovementioned changes by reversing the oxidant-antioxidant imbalance during $\mathrm{AC}$-induced hyperammonemia [39].

The antioxidant activity of MC extracts may vary, depending on the method of extraction. Wei and coworkers have shown that the free radical scavenging activity of MC extract was significantly increased by using heat drying process for extraction. The extract was found to exert higher proliferation activity on NIT-1 beta cells, thus resulting in possibly better antidiabetic activity of the fruit [15]. Liu et al. developed sulfated polysaccharide from MC with variable degrees of sulfation. There results showed that polysaccharides with high degree of sulfation exhibited better antioxidant activities as compared to native polysaccharides from MC in vitro [28].

Different ingredients in MC have found to be responsible for its potential antioxidant and antidiabetic properties. Lin et al. in 2011 isolated a number of new compounds including a novel cucurbitane-type triterpene glycoside, taiwacin A, taiwacin B, a known cucurbitane-type triterpene glycoside, and a known steroid glycoside from the stems and fruits of MC. They further proposed the structure of the new compounds by utilizing spectroscopic methods. All four compounds were shown to exhibit ABTS radical cation scavenging activity with IC50 values of $119.1 \pm 4.3,204.5 \pm 1.2$, $159.7 \pm 11.0$, and $98.1 \pm 2.4 \mu \mathrm{M}$ respectively [40]. In a study comparing the antioxidant activities of various herbal agents, the highest free radical scavenging activity was observed with the extracts of MC and Eugenia jambolana [41]. Kumar et al. also carried out the evaluation of the antioxidant activity of the total aqueous extract (TAE) and total phenolic extract (TPE) of MC fruits. The antioxidant activities were measured by using radical-scavenging methods. Further, the cytoprotective effects of the extracts on hydrogen peroxide $\left(\mathrm{H}_{2} \mathrm{O}_{2}\right)$ - and hypoxanthin-xanthin oxidase (HX-XO)-induced damage to various cell types were also measured. At 200 and $300 \mu \mathrm{g} /$ $\mathrm{mL}$, TPE showed dose-dependent cytoprotection against the used oxidants [42]. Dhar et al. have studied the in vitro antioxidant activity of MC seed oil containing conjugated octadecatrienoic fatty acid and alpha-eleostearic acid. Alphaeleostearic acid also decreased the lipid peroxidation level in a dose-dependent manner [43]. 
Table 1 Nutritional values of Momordica charantia raw leafy tips, raw pods, and their cooked forms

\begin{tabular}{|c|c|c|c|c|c|}
\hline Nutrients & & Leafy tips, raw & $\begin{array}{l}\text { Leafy tips, cooked, } \\
\text { boiled, drained, no salt }\end{array}$ & Pods, raw & $\begin{array}{l}\text { Pods, cooked, boiled, } \\
\text { drained, no salt }\end{array}$ \\
\hline \multirow[t]{7}{*}{ Proximates } & Water $(\mathrm{g})$ & 89.25 & 88.69 & 94.03 & 93.95 \\
\hline & Energy (Kcal) & 30 & 34 & 17 & 19 \\
\hline & Proteins (g) & 5.30 & 3.60 & 1 & 0.84 \\
\hline & Total lipids (fats) (g) & 0.69 & 0.20 & 0.17 & 0.18 \\
\hline & Carbohydrate, by difference (g) & 3.29 & 6.68 & 3.70 & 4.32 \\
\hline & Fiber, total dietary $(\mathrm{g})$ & - & 1.9 & 2.8 & 2.0 \\
\hline & Sugars, total & & 1.04 & & 1.95 \\
\hline \multirow[t]{7}{*}{ Minerals } & Calcium, Ca (mg) & 84 & 42 & 19 & 9 \\
\hline & Iron, $\mathrm{Fe}(\mathrm{mg})$ & 2.04 & 1.02 & 0.43 & 0.38 \\
\hline & Magnesium, Mg (mg) & 85 & 94 & 17 & 16 \\
\hline & Phosphorus, P (mg) & 99 & 77 & 31 & 36 \\
\hline & Potassium, K (mg) & 608 & 602 & 296 & 319 \\
\hline & Sodium, $\mathrm{Na}$ (mg) & 11 & 13 & 5 & 6 \\
\hline & Zinc, Zn (mg) & 0.30 & 0.30 & 0.80 & 0.77 \\
\hline \multirow[t]{10}{*}{ Vitamins } & Vitamin C, total ascorbic acid (mg) & 88 & 55.6 & 84.0 & 33.0 \\
\hline & Thiamin (mg) & 0.181 & 0.147 & 0.040 & 0.051 \\
\hline & Riboflavin (mg) & 0.362 & 0.282 & 0.040 & 0.053 \\
\hline & Niacin (mg) & 1.110 & 0.995 & 0.040 & 0.280 \\
\hline & Vitamin B-6 (mg) & 0.803 & 0.760 & 0.043 & 0.041 \\
\hline & Folate, DFE $(\mu \mathrm{g})$ & 128 & 88 & 72 & 51 \\
\hline & Vitamin A, RAE ( $\mu \mathrm{g})$ & 87 & 121 & 24 & 6 \\
\hline & Vitamin A, IU (IU) & 1734 & 2416 & 471 & 113 \\
\hline & Vitamin E (mg) & & 1.45 & & 0.14 \\
\hline & Vitamin $\mathrm{K}(\mu \mathrm{g})$ & & 163.1 & & 4.8 \\
\hline \multirow[t]{3}{*}{ Lipids } & Fatty acids, total saturated (g) & & 0.032 & & 0.014 \\
\hline & Fatty acids, total monounsaturated (g) & & 0.005 & & 0.033 \\
\hline & Fatty acids, total polyunsaturated (g) & & 0.083 & & 0.078 \\
\hline
\end{tabular}

The detailed nutritional information can be found from the United States Department of Agriculture, Agricultural research services websites at the following weblinks. For leafy tips, raw: http://ndb.nal.usda.gov/ndb/foods/show/2867; for pods, raw: http://ndb.nal.usda.gov/ndb/foods/show/2869; for pods, cooked, boiled, drained, without salt: http://ndb.nal.usda.gov/ndb/foods/show/2870

The antioxidant and $\alpha$-glucosidase inhibitory activity of the methanolic and ethyl acetate extracts of MC was studied by Sulaiman et al. The reducing power was found to be $692.56 \pm 43.38$ and $221.97 \pm 17.20 \mathrm{mM} \mathrm{AscAE/g} \mathrm{extract} \mathrm{re-}$ spectively, and the $\alpha$-glucosidase inhibitory activities were $18.04 \pm 0.47$ and $66.64 \pm 2.94 \%$, respectively [44]. Ching et al. evaluated the effects of maternal high-fructose intake on the offspring and if metabolic control in the offspring could improve from supplementing bioactive food components to the maternal diet. Their results showed that MC supplementation to dams could offset the adverse effects arising from the maternal high-fructose intake in adult offspring [45]. Tripathi and Chandra further studied the antihyperglycemic and antioxidative activities of $\mathrm{MC}$ aqueous extracts and found that it improved fasting blood glucose levels in rats. In addition, antioxidant activities such as the levels of superoxide dismutase, catalase, glutathione, and glutathione-s-transferase within the heart, kidney, and liver tissues and TBARS levels were also improved [46]. These authors also studied the effect of $\mathrm{MC}$ on antioxidant levels and lipid peroxidation in heart tissue of non-diabetic and alloxan-induced diabetic rats. They found that MC treatment reduced the elevated levels of fasting blood glucose, decreased lipid peroxidation and increased antioxidant enzyme activity in heart tissue of diabetic rats [47]. These results all suggest that some portion of the antidiabetic benefits of $\mathrm{MC}$ also come from its antioxidative properties, which supplement the hypoglycemic effects with the reversal of oxidantinduced damage.

Further studies in diabetic animal models showed that MC extracts possess not only the hypoglycemic properties but can also alleviate diabetes-related adverse effects. A study by Chaturvedi and George in alloxan-induced diabetic rats subjected to a sucrose load showed that MC maintained the 
normal glucose levels in all experimental groups. It also reduced triglyceride and low-density lipoprotein levels and increased high-density lipoprotein levels. It further improved the antioxidant status by lowering the levels of thiobarbituric acid-reactive substances and normalizing the levels of reduced glutathione [48]. Teoh et al. studied the nephro protective effect of MC extract in streptozotocin-induced diabetes in rats. The MC extract was found to reverse streptozotocin-induced thickening of the basement membrane of the Bowman's capsule, edema, and hypercellularity of the proximal tubules, necrosis, and hyaline deposits. The MC extract also exerted nephro-protective activity by preventing the oxidative damage involved in the diabetic kidney [49]. In a separate study, the livers of the diabetic rats had hepatocytes showing features of inflammation. These signs of liver damage were found to reverse with administration of the MC extract [50].

Another important study was to determine the potential cell reparative effects of MC boiling water extract (MCE) on the HIT-T15 Hamster Pancreatic beta cells by Xiang and colleagues. The high molecular weight fraction of MCE (MHMF, MW $>3 \mathrm{kDa}$ ) exhibited better repairing in alloxan-damaged cells than the low molecular weight fraction (MLMF, $\mathrm{MW}<\mathrm{or}=3 \mathrm{kDa}$ ). Contrary to the cell proliferation improvements, it was observed that MLMF showed higher overall activity through the increase in insulin secretion of both normal and damaged cells. Their results indicated MCE has significant repairing activity against superoxide anion radicals. Further, their data shows that different components of the extracts may elicit different aspects of response when it comes to its antidiabetic properties [51]. Antioxidant activities of the aqueous extract of seeds of two varieties of MC (MCSEt1 and MCSEt2) were studied in streptozotocin-induced diabetes in rats. The extracts were shown to possess protective effects against lipid peroxidation by scavenging of free radicals, thus reducing the risk of diabetic complications. The effect was more pronounced in MCSEt1 compared to MCSEt2 [52].

Kavitha and colleagues evaluated the effects of ethanolic fruit extract of $\mathrm{MC}$ on stress-induced changes in Wister albino rats. MC pretreatment significantly countered acute stress-induced changes in plasma corticosterone levels and brain monoamine levels (5-hydroxy tryptamine, norepinephrine, epinephrine, and dopamine) [53]. Nerurkar et al. have also investigated the neuroprotective effects of $\mathrm{MC}$ on high-fat diet (HFD)-associated changes in C57BL/ 6 female mice. MC treatment ameliorated HFD-associated changes in BBB permeability. The treatment also normalized HFD-induced glial cells activation and expression of neuroinflammatory markers such as NF-kB1, IL-16, IL-22 as well as IL-17R in brains of MC-dosed mice. Brain oxidative stress was significantly reduced by $\mathrm{MC}$ along with reduction in FoxO, normalization of Sirtl protein expression, and upregulation of Sirt3 mRNA expression [54].
The protective effects of MC extract on lipid peroxidation induced by immobilization stress in rats were studied. Here too, MC inhibited stress-induced lipid peroxidation in rats, which was in part due to increasing levels of reduced glutathione and increased catalase activity [55]. The anti-oxygenic activity of MC pulp and seed powders as well as their various solvent extracts were evaluated. Overall, the MC pulp and seed powders exhibited stronger anti-oxygenic activity than other solvent extracts. However, MC pulp and its extracts showed little higher activity when compared to MC seeds and its extracts [56]. De et al. determined the effects of fresh juices of MC and tomato separately as well as together in repairing DNA damage. They found that the combination could protect from DNA damage, but not as effectively as either agent alone [57].

\section{Anti-inflammatory Activity}

Immune cells are involved in inflammation-based responses. Bitter melon is known to interact with immune cells. Multiple studies have shown that various extracts of MC can act as immunomodulators and, hence, can also act as antiinflammatory agents. Bao et al. looked at the effects of MC treatment in C57BL/6 mice administered with HFD. Once again, MC-containing diets reduced the HFD-induced obesity as well as insulin resistance. MC was also shown to decrease macrophage infiltration into epididymal adipose tissues (EAT) and brown adipose tissues (BAT). MC further reduced IL- 6 and TNF- $\alpha$ expression in EAT and normalized serum levels of cytokines, suggesting the potential antiinflammatory role of MC in obese rats [58]. Further, $\mathrm{Xu}$ et al. studied the effects of $\mathrm{MC}$ on mitochondrial function during the development of obesity-linked fatty liver in C57BL/6 mice. The mouse model involved feeding with high fat diet (HFD) supplemented with two doses of MC powder $\{0.5$ $(\mathrm{HFD}+0.5 \mathrm{MC})$ and $5(\mathrm{HFD}+5 \mathrm{MC}) \mathrm{g} / \mathrm{kg}\}$. The mice in $\mathrm{HFD}+5 \mathrm{MC}$ group showed less body and tissue weight gain and less hyperglycemia and hyperlipidemia compared to control HFD group. In both treatment groups, serum interleukin-6 concentration was lower than that in HFD group, though the serum C-reactive protein concentration was lower only in $\mathrm{HFD}+5 \mathrm{MC}$ group [59].

Hsieh et al. suggested that the anti-adiposity effects of MC seed oil is linked to white adipose tissue delipidation, inflammation, and browning [60].

The triterpene, EMCD extracted from wild variant of MC WB24 have been shown to suppress TNF $\alpha$-induced expression of inflammatory markers including inducible nitric oxide synthase (iNOS), NF-kB, protein-tyrosine phosphatase-1B, and interleukin-1 $\beta$ in FL83B cells. Moreover, EMCD exerted more pronounced anti-inflammatory activity in combination with EGCG [61]. 
The protective effect of MC in tibial and sural nerve transection (TST)-induced neuropathic pain were studied in rats. TST induce significant development of cold allodynia, mechanical and heat hyperalgesia, dynamic mechanical allodynia, and functional deficit in walking along with increase in TBARS and TNF- $\alpha$ levels. Administration of MC at various doses significantly reduced TST-induced behavioral and biochemical changes [62]. Hsu et al. showed that ethyl acetate extract of MC fruit and its saponofiable as well as nonsaponifiable fractions reduced pro-inflammatory cytokine and matrix metalloproteinase (MMP)-9 levels in Propionibacterium acnes-stimulated THP-1 cells. The intradermal injection of MC extract in mice effectively attenuated $P$. acnes-induced ear swelling and granulomatous inflammation [63].

In further studies, Lii and group have shown that the ethanol extracts of MC exerted the greatest decrease of lipopolysaccharide treatment (LPS)-induced production of nitric oxide and prostaglandin E2 as well as iNOS and pro-interleukin1 beta expression in RAW264.7 macrophages [64]. Similarly, Kobori et al. also showed that the butanol-soluble fraction of MC extract decreased LPS-induced TNF- $\alpha$ production in RAW 264.7 cells [65].

\section{Anticancer Activities}

In the previous three decades, the research with $\mathrm{MC}$ was focused on its antidiabetic, anti-inflammatory, and antioxidative properties. Over the last 10 years, the interest in studying MC in the prevention or treatment of cancer has increased. The Warburg effect, which deals with the shift in metabolic profile of tumor cells by increasing their dependence on glycolysis and lactic acid fermentation, also regained prominence during this time. $\mathrm{MC}$ was found to be effective in multiple cancers by affecting multiple pathways including metabolic pathways. In the following section, we have summarized the potential activities of $\mathrm{MC}$ and its chemical constituents against numerous cancers including breast, colon, pancreatic, liver, prostate, and skin cancers.

\section{Breast Cancer}

Weng et al. have studied a cucurbitane-type triterpene DMC isolated from wild MC. DMC was found to activate PPAR- $\gamma$. The pharmacological inhibition of PPAR- $\gamma$ protected cells from DMC's anti-proliferative effect, further confirming its mechanism of action. DMC suppressed the expression of PPAR- $\gamma$ targets, such as cyclin D1, CDK6, Bcl-2, XIAP, cyclooxygenase-2, NF- $\kappa \mathrm{B}$, and estrogen receptor- $\alpha$. It induced ER stress, as manifested by GADD153 and GRP78 induction. DMC also inhibited mTOR-p70S6K signaling by downregulating Akt and activating AMPK. The study suggested involvement of PPAR- $\gamma$ signaling in the antitumor activity of MC [66].
Fang et al. have isolated and characterized a $14-\mathrm{kDa}$ ribonuclease (RNase MC2) from MC seeds. RNase MC2 showed both cytostatic and cytotoxic properties against MCF-7 breast cancer (BC) cells causing nuclear damage, leading to either early or late apoptosis. Additionally, RNase MC2 induced activation of MAPKs such as p38, JNK, and ERK as well as Akt, caspases 8, 9, and 7, enhanced Bax and cleaved PARP levels, further contributing to apoptosis [67].

In another study, the anticancer activity of $\mathrm{MC}$ extract was studied in MCF-7 and MDA-MB-231 human BC cells as well as in primary human mammary epithelial cells. MC treatment reduced cell proliferation and induced apoptosis in $\mathrm{BC}$ cells tested, which was accompanied by increased levels of cleaved poly (ADP-ribose) polymerase and caspase activation. The treatment also inhibited the expression of survivin and claspin. MC-treated MCF-7 cells accumulated during the G2-M phase of the cell cycle. MC treatment enhanced p53, p21, and pChk1/2 expression and inhibited cyclin B1 and cyclin D1 expression, suggesting a further mechanism involving cell cycle regulation [68]. Nagasawa et al. have shown that SHN virgin mice supplimented with free access to hot water extract of MC $(0.5 \%)$ in drinking water developed significantly less breast tumors. The treatment also inhibited uterine adenomyosis. Moreover, there were no adverse effects observed with MC treatment [69].

Eleostearic acid ( $\alpha$-ESA) is a conjugated form of linolenic acid (CLN) that constitutes up to $60 \%$ of MC seed oil. Grossmann and group have studied the effects of $\alpha$-ESA on estrogen receptor (ER)-negative MDA-MB-231 (MDA-wt) and ER-positive MDA-ERalpha7 human BC cells. Their studies found that $\alpha$-ESA inhibited proliferation of MDA-wt and MDA-ERalpha7 cells. $\alpha$-ESA treatment also induced apoptosis in both cell lines ( $70 \%-90 \%)$, whereas CLN resulted in only 5 to $10 \%$ apoptosis. Moreover, the addition of $\alpha$-ESA also caused loss of mitochondrial membrane potential and translocation of apoptosis-inducing factor as well as endonuclease $\mathrm{G}$ from the mitochondria to the nucleus. $\alpha$-ESA treatment also caused a G(2)-M arrest in the cell cycle [70].

Lee-Huang et al. have studied the efficacy of a peptide MAP30 found in MC on estrogen-independent and highly metastatic human breast tumor MDA-MB-231. The treatment resulted in inhibition of cancer cell proliferation and inhibition of HER2 gene expression in vitro. When MDA-MB-231 cells were transferred into SCID mice, they developed extensive metastases and all mice succumbed to tumor by day 46 . Treatment of the BC-bearing mice with MAP30 injections resulted in significant increases in survival, with $20-25 \%$ of the mice remaining tumor free for 96 days [71].

\section{Colon Cancer}

The utilization of multiple food agents with potential cancer preventive properties in the daily diet has been attributed to 
relatively lower incidence rate of colon cancer in South and East Asian countries. MC is one such food, and hence, it has also been studied for its potential role in preventing/treating colon cancer. We have evaluated the potential of methanolic extracts of MC (whole fruit and skin) on colon cancer stem and progenitor cells. Both the whole fruit and the skin extracts significantly inhibited cell proliferation and colony formation, where the whole fruit extract showed greater efficacy. In these studies, the cells were arrested at the S-phase of cell cycle. The extracts induced the cleavage of LC3B, but not caspase $3 / 7$, indicating that the cells were undergoing autophagy and not apoptosis. Autophagy was further confirmed by reduced Bcl2 and increased Beclin-1, Atg 7, and 12 expression after MC treatment. The treatment also led to reduced cellular ATP levels and activation of AMPK. Exogenous additions of ATP led to revival of cell proliferation even in the presence of MC. Treatment with MC resulted in decrease in the number, and size of colonospheres coupled with decreased expression of DCLK1 and Lgr5 suggested the activity of extracts on colon cancer stem cells [3]. The effects of the same MC extract on anticancer activity and bioavailability of doxorubicin (DOX) was also studied. MC extract enhanced the effect of DOX on proliferation and sensitized the cancer cells toward DOX upon pretreatment. This synergism was attributed to both an increase in drug uptake and reduction in drug efflux. Reduced expression of multidrug resistance conferring proteins (MDRCP) P-glycoprotein, MRP-2, and BCRP was also observed. Suppression of DOX efflux in MDCK cells overexpressing the three MDRCP individually suggests that MC extract is a potent inhibitor of MDR function. MC extract also suppressed PXR (a xenobiotic sensing nuclear receptor that controls the expression of MDRCP) promoter activity. These results suggested that $\mathrm{MC}$ can enhance efficacy of conventional cancer chemotherapy [4].

$\mathrm{Li}$ et al. also showed that the methanolic extracts of MC exerted cytotoxic activity on Hone-1 (nasopharyngeal carcinoma), AGS (gastric adenocarcinoma), HCT-116 (colorectal carcinoma), and CL1-0 lung adenocarcinoma in a timedependent manner. MC treatment induced apoptosis by activation of caspase-3, enhanced cleavage of downstream DFF45 and PARP, resulting in DNA fragmentation and nuclear condensation. Bax expression was increased, whereas Bcl2 was decreased in all treated cells, indicating the involvement of mitochondrial pathway in MC activity [72]. Another study identified a resistance-like protein P-B from MC by using high-speed counter-current chromatography (HSCCC) coupled with a reverse micelle solvent system. Fractions I and III were identified as resistance-like protein P-B and pentatricopeptide repeat-containing protein, respectively. However, fraction II, which is thought to be a new protein, has not yet been identified. The fraction II had excellent anticancer activity (IC50 value $0.116 \mathrm{mg} / \mathrm{ml}$ for $48 \mathrm{~h}$ treatment) on human gastric cancer cell line SGC-7901 [73].
Kupradinun et al. examined effect of MC against clastogens, cyclophosphamide (CYP), and DMBA using in vivo erythrocyte micronucleus assay in mice and azoxymethane (AOM)-induced colon carcinogenesis in rats. The study demonstrated that mice fed with MC containing diets showed potential anticancer activity against clastogens. Surprisingly, the MC extracts did not show any preventive effects in AOM model with treatment resulting in increased incidence and multiplicities of colon tumors than control [74]. Fan et al. showed that proliferation of LoVo cells was suppressed by MAP30 in both time- and dose-dependent manner. MAP30-induced the apoptotic nuclei observed in LoVo cells. Additionally, genomic degradation was detected in treated cells by using single-cell gel electrophoresis (comet assay). Upregulation of Bax and downregulation of Bcl-2 were also observed [75]. Yasui et al. investigated the effects of free fatty acids prepared from the MC seed oil in Caco-2 cells. The expression of $\mathrm{Bcl}-2$ was found to be decreased by the treatment. Apoptosis regulators GADD45 and p53 were also remarkably upregulated by the treatment [76].

Konishi et al. observed that MC fraction extracted with $40 \%$ methanol showed the greatest increase of rhodamine123 accumulation in Caco-2 cells. Inhibitory compound in the MC fraction was identified as 1-monopalmitin. They hypothesized that that certain types of monoglyceride might modulate the bioavailability of drugs by inhibiting efflux mediated by P-gp, which matched the MDR-based claims of Kwatra et al. [77]. The inhibitory activity of 1-monopalmitin and related compounds suggested that the P-gp inhibition activity was independent of degree of unsaturation of fatty acid instead depending on chain length, with the monoglyceride structure deciding the ultimate inhibition of P-gp efflux. Monoglycerides could therefore alter drug pharmacokinetics by inhibiting efflux [78].

Deep et al. tested MC fruit extract $(2.5 \%$ and $5 \%$ of standard mice feed) against 3,4 benzo(a)pyrene [B(a)P]-induced forestomach papillomagenesis in Swiss albino mice. A significant decrease in tumor burden was observed after MC treatment. Also, total tumor incidence reduced by $80 \%$ to $90 \%$ in short-term treatment and close to $75 \%$ in long-term treatment [79]. Kohno et al. studied the effects of MC containing diet in AOM-induced colonic neoplasms in male F344 rats. The treatment reduced both the incidence and the multiplicity of colonic adenocarcinoma. The inhibition was associated with increased content of CLN in colonic mucosa and liver [80]. In another study, Kohno et al. had studied the dietary effects of CLN isolated from the seeds of MC against AOM - induced colonic aberrant crypt foci (ACF) in male F344. Dietary administration of CLN caused a significant reduction in the frequency of ACF, lowered the PCNA index, and induced apoptosis in ACF. The possible mechanism of chemo-preventive activity was hypothesized to be modulation of cryptal cell proliferation activity and/or apoptosis [81]. 


\section{Pancreatic Cancer}

The efficacy of MC juice against pancreatic carcinoma cells both in culture and MiaPaCa-2 tumors in nude mice was investigated. Their results showed that MC $(2-5 \% v / v)$ decreased cell viability in all tested cell lines by inducing strong apoptotic cell death caused by activation of caspases. It was also found to activate AMPK, alter expression of Bcl-2 family members, and release cytochrome-c into the cytosol. Additionally, the treatment with $\mathrm{MC}$ juice resulted in reduced survivin and XIAP expression but increased p21, CHOP, and phosphorylated MAP kinases levels. Oral administration of lyophilized MC juice inhibited MiaPaCa-2 tumor xenograft growth by $60 \%$ without noticeable toxicity in nude mice. IHC analyses of xenografts suggested that MC also inhibits proliferation, induces apoptosis, and activates AMPK in mouse model [82].

\section{Liver Cancer}

Fang et al. have shown that RNase MC2 restricted cell proliferation and induced apoptosis in HepG2 cells. RNase MC2 caused S-phase cell cycle arrest, induced phosphorylation of ERK and JNK as well as caused activation of both caspase- 8 and caspase-9 mediated apoptosis. RNase MC2 treatment also reduced Bcl-2 and enhanced Bak expression. RNase MC2 suppressed tumor growth by inducing apoptosis in the HepG2 xenograft-bearing nude mice. RNase MC2 mediated apoptosis was confirmed by enhanced number of caspase-3-, PARP-, and TUNEL-positive cells in the tumor tissues of HepG2 xenograft-bearing nude mice [83]. Further studies have been performed to establish the efficacy of MAP30 against human hepatocellular carcinoma HepG2 cells and HepG2-bearing mice. The mechanistic studies showed the involvement of both extrinsically (caspase-8) as well as intrinsically (caspase-9) induced apoptosis in MAP30-treated cells. The peptide was also found to be effective in HepG2 tumorbearing nude mice [84].

\section{Prostate Cancer}

$\mathrm{Ru}$ et al. have evaluated the of $\mathrm{MC}$ against human prostate cancer (PC3 and LNCaP) cells. The cancer cells treated with $\mathrm{MC}$ extract got arrested during the S-phase of the cell cycle. MC treatment also modulated cyclin D1, cyclin E, and p21 expression within the cells. The treatment also increased Bax expression and induced PARP cleavage. Oral gavage of MC extract in TRAMP mice delayed the progression to high-grade prostatic intraepithelial neoplasia by $31 \%$. Prostate tissue from MC extract-fed mice displayed approximately $51 \%$ reduction in the expression of proliferating cell nuclear antigen (PCNA) [85].
Pitchakarn et al. have shown that MC leaf extract and Kuguacin $\mathrm{J}(\mathrm{KuJ})$ exerted growth inhibition through a G1arrest of cell cycle and induced apoptosis in androgendependent LNCaP cells. MC leaf extract and $\mathrm{KuJ}$ also reduced the expression levels of prostate-specific antigen (PSA) and androgen receptor (AR). These two together induced P53 protein level upon treatment. Additionally, the inhibition of p53 by RNAi confirmed the mechanism partially to be p53-dependent. MC leaf extract and $\mathrm{KuJ}$ were less toxic in normal prostate cells (PNT1A); hence, these effects were prostate cancer specific [86]. In another study, the same group studied the anti-invasive effects of $\mathrm{MC}$ on a rat prostate cancer cell line (PLS10) and found that MC leaf extract significantly reduced the migration and invasion ability of cells in vitro. The MC extract successfully inhibited MMP-2, MMP-9, and uPA secretion from PLS10. It also markedly increased the mRNA expression of TIMP-2. The collagenase type IV activity was also partially inhibited by MC leaf extract. Intravenous inoculation of PLS10 to nude mice resulted in $20 \%$ deaths, but a $100 \%$ survival rate in the mice given MC leaf extract. The treatment did not affect the incidence rate of lung metastasis but reduced the percentage of lung area occupied by metastatic lesions [87].

Xiong et al. also isolated a protein from MC seeds, which they called MCP30. MCP30 - induced apoptosis in PIN and PCa cells in vitro and also suppressed the PC-3 growth in vivo with minimal effects on normal prostate cells. MCP30 inhibited HDAC-1 activity and promoted histone- 3 and histone-4 protein acetylation. The treatment also induced PTEN expression in both cell lines, resulting in inhibition of Akt phosphorylation. MCP30 inhibited Wnt signaling by reduced nuclear accumulation of $\beta$-catenin and decreased the levels of c-Myc and Cyclin-D1 [88].

\section{Skin Cancer}

Agrawal and Beohar have shown that the treatment with MC fruit and leaf extract in Swiss albino mice resulted in reduced tumor incidence, tumor burden, and cumulative number of papillomas when compared to untreated controls. In a melanoma model, the mice receiving either fruit or leaf extracts showed improved life spans and reduced tumor volumes [89]. Further, Ganguly et al. have also evaluated the anticarcinogenic effect of aqueous extract of MC fruit in skin carcinogenesis mouse model. Though high doses of extract had an adverse effect on the health of the animals, but on reducing the dose by half, the extract reduced the development of skin tumors and increased life expectancy. Hence, this study showed both the potential positive effects and potential toxic effects upon overdosing [90].

Singh et al. have studied the potential of different MC plant parts (peel, pulp, seed, and whole fruit) extracts on mouse model of skin papillomagenesis. Topical use of MC against 
DMBA resulted in reduced incident rate, tumor burden, and cumulative number of papillomas. The results suggested that the most effective extract was the MC peel, indicating that biotransformation system enzymes may be the reason for these effects [91].

\section{Miscellaneous Cancers}

MC has been evaluated in other cancers including cervical cancer [92, 93], leukemia [94-97], adrenocortical cancer [98], adenocarcinoma of rat prostate [99], human lung adenocarcinoma cells with different metastatic ability [100], human nasopharyngeal carcinoma CNE2 and HONE1 cells [101], and head and neck squamous cell carcinoma cells [102].

\section{Conclusions}

MC, also called as bitter melon, an edible fruit, is traditionally used in alternative system of medicines as a remedy for treatment of diabetes, laxative, antiulcer, anthelmintic, and antimalarial activity. MC contains numerous important chemical constituents that thought to be involved in therapeutic activity of MC. Several studies have been conducted to establish therapeutic activities of MC for various disorders. MC has exhibited multiple targeting ability against several cancers in vitro and animal models, but systematic clinical studies are needed to establish its efficacy in patients. More studies are also needed to establish its detailed pharmacological and toxicological profile.

\section{Compliance with Ethical Standards}

Conflict of Interest Deep Kwatra, Prasad Dandawate, Subhash Padhye, and Shrikant Anant declare that they have no conflict of interest.

Human and Animal Rights and Informed Consent This article does not contain any studies with human or animal subjects performed by any of the authors.

\section{References}

1. Organization WH. WHO traditional medicine strategy 20022005. 2002.

2. Patwardhan B et al. Ayurveda and traditional Chinese medicine: a comparative overview. Evidence-Based Complement Alternat Med. 2005;2(4):465-73.

3. Kwatra D et al. Methanolic extracts of bitter melon inhibit colon cancer stem cells by affecting energy homeostasis and autophagy. Evidence-Based Complementary and Alternative Medicine, 2013. 2013.

4. Kwatra D et al. Bitter melon extracts enhance the activity of chemotherapeutic agents through the modulation of multiple drug resistance. J Pharm Sci. 2013;102(12):4444-54.
5. Nkambo W, Anyama NG, Onegi B. In vivo hypoglycemic effect of methanolic fruit extract of Momordica charantia L. Afr Health Sci. 2013;13(4):933-9.

6. Vyas A et al. Perspectives on new synthetic curcumin analogs and their potential anticancer properties. Curr Pharm Des. 2013;19(11):2047.

7. Zhang D-W. Curcumin and diabetes: A systematic review. evidence-based complementary and alternative medicine, 2013. 2013.

8. Panaskar $\mathrm{SN}$ et al. Aegle marmelos Correa leaf extract prevents secondary complications in streptozotocin-induced diabetic rats and demonstration of limonene as a potent antiglycating agent. J Pharm Pharmacol. 2013;65(6):884-94.

9. Subramaniam D et al. Activation of apoptosis by 1-hydroxy-5, 7dimethoxy-2-naphthalene-carboxaldehyde, a novel compound from Aegle marmelos. Cancer Res. 2008;68(20):8573-81.

10. Heiser CB. The gourd book. 1993: University of Oklahoma Press.

11. Tindall HD. Vegetables in the tropics. Macmillan Press Ltd, 1983.

12. Reyes M, Gildemacher B, Jansen G. Momordica L. Wageningon. Netherland: Pudoc Scientific Publishers; 1994.

13. Marr KL, Mei XY, Bhattarai NK. Allozyme, morphological and nutritional analysis bearing on the domestication of Momordica charantia L. (Cucurbitaceae). Econ Bot. 2004;58(3):435-55.

14. Grover J, Yadav S. Pharmacological actions and potential uses of Momordica charantia: a review. J Ethnopharmacol. 2004;93(1): 123-32.

15. Wei $\mathrm{L}$ et al. Increase in the free radical scavenging capability of bitter gourd by a heat-drying process. Food Funct. 2013;4(12): $1850-5$.

16. Zhu $\mathrm{Y}$ et al. Effect of superfine grinding on antidiabetic activity of bitter melon powder. Int J Mol Sci. 2012;13(11):14203-18.

17. Chao $\mathrm{C}-\mathrm{Y}$ et al. Anti-inflammatory effect of Momordica Charantia in sepsis mice. Molecules. 2014;19(8):12777-88.

18. Costa JGM et al. Antibacterial activity of Momordica charantia (Curcubitaceae) extracts and fractions. J Basic Clin Pharma. 2010;2(1):45.

19. Santos KK et al. Trypanocide, cytotoxic, and antifungal activities of Momordica charantia. Pharm Biol. 2012;50(2):162-6.

20. Pongthanapisith $\mathrm{V}$ et al. Antiviral protein of Momordica charantia L. inhibits different subtypes of Influenza A. Evid Based Complement Alternat Med. 2013;2013:729081.

21. Fang FE, Ng TB. Bitter gourd (Momordica charantia) is a cornucopia of health: a review of its credited antidiabetic, anti-HIV, and antitumor properties. Curr Molec Med. 2011;11(5):417-36.

22. Lal $\mathrm{J}$ et al. In vitro anthelmintic action of some indigenous medicinal plants on Ascardia galli worms. Indian J Physiol Pharmacol. 1975;20(2):64-8.

23. Frame AD et al. Plants from Puerto Rico with anti-Mycobacterium tuberculosis properties. P R Health Sci J. 1998;17(3):243-52.

24. Ojewole J, Adewole SO, Olayiwola G. Hypoglycaemic and hypotensive effects of Momordica charantia Linn (Cucurbitaceae) whole-plant aqueous extract in rats. Cardiovasc J South Africa: Off J Southern Africa Cardiac Soc South African Soc Cardiac Practitioners. 2005;17(5):227-32.

25. Shih C-C, Lin C-H, Lin W-L. Effects of Momordica charantia on insulin resistance and visceral obesity in mice on high-fat diet. Diabetes Res Clin Pract. 2008;81(2):134-43.

26. Deng Y-Y et al. Immunomodulatory activity and partial characterisation of polysaccharides from Momordica charantia. Molecules. 2014;19(9):13432-47.

27. Manik S, Gauttam V, Kalia A. Anti-diabetic and antihyperlipidemic effect of allopolyherbal formulation in OGTT and STZ-induced diabetic rat model. 2013.

28. $\mathrm{Lu} \mathrm{KH}$ et al. Wild bitter gourd protects against alcoholic fatty liver in mice by attenuating oxidative stress and inflammatory responses. Food Funct. 2014;5(5):1027-37. 
29. Malik ZA, Singh M, Sharma P. Neuroprotective effect of Momordica charantia in global cerebral ischemia and reperfusion induced neuronal damage in diabetic mice. J Ethnopharmacol. 2011;133(2):729-34.

30. Haque ME, Alam MB, Hossain MS. The efficacy of cucurbitane type triterpenoids, glycosides and phenolic compounds isolated from Momordica charantia: a review. IJPSR. 2011;2(5):1135-46.

31. Pitrat M, Chauvet M, Foury C. Diversity, history and production of cultivated cucurbits. in I International Symposium on Cucurbits 492, 1997.

32. Decker-Walters DS. Cucurbits, sanskrit, and the Indo-Aryas 1. Econ Bot. 1999;53(1):98-112.

33. Behera $\mathrm{T}$ et al. Morphological and molecular analyses define the genetic diversity of Asian bitter gourd ('Momordica charantia'L.). 2012.

34. Satyavati G, Raina M, Sharma M. Medicinal plants of India. vol. 1. New Delhi: Indian council of medical research; 1976.

35. Yeşilada E, Gürbüz II, Sh H. Screening of Turkish antiulcerogenic folk remedies for anti-Helicobacter pylori activity. J Ethnopharmacol. 1999;66(3):289-93.

36. Chakravarty H. Cucurbits of India and their role in the development of vegetable crops. Biology and utilization of the cucurbitaceae. Ithaca: Comstock, Cornell University Press; 1990.

37. Behera $\mathrm{T}$ et al. Minor cucurbits. genetics, genomics and breeding of cucurbits, 2011: p. 17

38. Behera TK et al. 2 bitter gourd: botany, horticulture, breeding. Hortic Rev. 2010;37:101.

39. Thenmozhi AJ, Subramanian P. Antioxidant potential of Momordica charantia in ammonium chloride-induced hyperammonemic rats. Evidence-Based Complement Alternat Med, 2011. 2011.

40. Lin K-W, Yang S-C, Lin C-N. Antioxidant constituents from the stems and fruits of Momordica charantia. Food Chem. 2011;127(2):609-14.

41. Santos AK et al. Antioxidant activity of five Brazilian plants used as traditional medicines and food in Brazil. Pharmacogn Mag. 2010;6(24):335.

42. Kumar R et al. In vitro evaluation of antioxidants of fruit extract of Momordica charantia L. on fibroblasts and keratinocytes. J Agric Food Chem. 2010;58(3):1518-22.

43. Dhar $\mathrm{P}$ et al. Antioxidative effect of conjugated linolenic acid in diabetic and non-diabetic blood: an in vitro study. J Oleo Sci. 2007;56(1):19-24.

44. Sulaiman SF, Ooi KL. Antioxidant and $\alpha$-glucosidase inhibitory activities of cucurbit fruit vegetables and identification of active and major constituents from phenolic-rich extracts of Lagenaria siceraria and Sechium edule. J Agric Food Chem. 2013;61(42): 10080-90.

45. Ching R et al. Supplementation of bitter melon to rats fed a highfructose diet during gestation and lactation ameliorates fructoseinduced dyslipidemia and hepatic oxidative stress in male offspring. J Nutr. 2011;141:1664-72.

46. Tripathi UN, Chandra D. Anti-hyperglycemic and anti-oxidative effect of aqueous extract of Momordica charantia pulp and Trigonella foenum graecum seed in alloxan-induced diabetic rats. 2010.

47. Tripathi UN, Chandra D. The plant extracts of Momordica charantia and Trigonella foenum graecum have antioxidant and anti-hyperglycemic properties for cardiac tissue during diabetes mellitus. Oxidative Med Cell Longev. 2009;2(5): 290-6.

48. Chaturvedi P, George S. Momordica charantia maintains normal glucose levels and lipid profiles and prevents oxidative stress in diabetic rats subjected to chronic sucrose load. J Med Food. 2010;13(3):520-7.
49. Teoh S, Latiff AA, Das S. Histological changes in the kidneys of experimental diabetic rats fed with Momordica charantia (bitter gourd) extract. Rom J Morphol Embryol. 2010;51(1):91-5.

50. Teoh S, Latiff A, Das S. A histological study of the structural changes in the liver of streptozotocin-induced diabetic rats treated with or without Momordica charantia (bitter gourd). La Clinica Terapeutica. 2008;160(4):283-6.

51. Xiang L et al. The reparative effects of Momordica Charantia Linn. extract on HIT-T15 pancreatic beta-cells. Asia Pac J Clin Nutr. 2007;16(1):249-52.

52. Sathishsekar D, Subramanian S. Antioxidant properties of Momordica Charantia (bitter gourd) seeds on Streptozotocin induced diabetic rats. Asia Pac J Clin Nutr. 2005;14(2):153-8.

53. Kavitha CN, Babu SM, Rao MB. Influence of Momordica charantia on oxidative stress-induced perturbations in brain monoamines and plasma corticosterone in albino rats. Indian $\mathrm{J}$ Pharmacol. 2011;43(4):424.

54. Nerurkar PV et al. Momordica charantia (bitter melon) attenuates high-fat diet-associated oxidative stress and neuroinflammation. J Neuroinflammation. 2011;8(64):2094-8.

55. Chaturvedi P. Bitter melon protects against lipid peroxidation caused by immobilization stress in albino rats. Int J Vitam Nutr Res. 2009;79(1):48-56.

56. Padmashree A et al. Studies on the antioxygenic activity of bitter gourd (Momordica charantia) and its fractions using various in vitro models. J Sci Food Agric. 2011;91(4):776-82.

57. De S, Ganguly C, Das S. Natural dietary agents can protect against DMBA genotoxicity in lymphocytes as revealed by single cell gel electrophoresis assay. Teratog Carcinog Mutagen. 2003;23(S1): 71-8.

58. Bao B et al. Momordica charantia (Bitter Melon) reduces obesityassociated macrophage and mast cell infiltration as well as inflammatory cytokine expression in adipose tissues. PLoS One. 2013;8(12):e84075.

59. $\mathrm{Xu} \mathrm{J}$ et al. Bitter gourd inhibits the development of obesityassociated fatty liver in $\mathrm{C} 57 \mathrm{BL} / 6$ mice fed a high-fat diet. J Nutrit. 2014;144(4):475-83.

60. Hsieh $\mathrm{C}-\mathrm{H}$ et al. Altered white adipose tissue protein profile in C57BL/6J mice displaying delipidative, inflammatory, and browning characteristics after bitter melon seed oil treatment. PLoS One. 2013;8(9):e72917.

61. Cheng H-L et al. EMCD, a hypoglycemic triterpene isolated from Momordica charantia wild variant, attenuates TNF- $\alpha$-induced inflammation in FL83B cells in an AMP-activated protein kinaseindependent manner. Eur J Pharmacol. 2012;689(1):241-8.

62. Jain $\mathrm{V}$ et al. Antinociceptive and antiallodynic effects of Momordica charantia L. in tibial and sural nerve transectioninduced neuropathic pain in rats. Nutr Neurosci. 2014;17(2):88 96.

63. Hsu C et al. Wild bitter melon (Momordica charantia Linn. var. abbreviata Ser.) extract and its bioactive components suppress Propionibacterium acnes-induced inflammation. Food Chem. 2012;135(3):976-84.

64. Lii CK et al. Suppressive effects of wild bitter gourd (Momordica charantia Linn. var. abbreviata ser.) fruit extracts on inflammatory responses in RAW264.7 macrophages. J Ethnopharmacol. 2009;122(2):227-33.

65. Kobori $\mathrm{M}$ et al. Bitter gourd suppresses lipopolysaccharideinduced inflammatory responses. J Agric Food Chem. 2008;56(11):4004-11.

66. Weng $\mathrm{J}$ et al. Cucurbitane triterpenoid from Momordica charantia induces apoptosis and autophagy in breast cancer cells, in Part, through peroxisome proliferator-activated receptor gamma activation. Evid Based Complement Alternat Med. 2013.

67. Fang EF et al. RNase MC2: a new Momordica charantia ribonuclease that induces apoptosis in breast cancer cells associated with 
activation of MAPKs and induction of caspase pathways. Apoptosis. 2012;17(4):377-87.

68. Ray RB et al. Bitter melon (Momordica charantia) extract inhibits breast cancer cell proliferation by modulating cell cycle regulatory genes and promotes apoptosis. Cancer Res. 2010;70(5):1925-31.

69. Nagasawa H, Watanabe K, Inatomi H. Effects of bitter melon (Momordica charantia 1.) or ginger rhizome (Zingiber offifinale rosc) on spontaneous mammary tumorigenesis in SHN mice. Am J Chin Med. 2002;30(02n03):195-205.

70. Grossmann ME et al. Eleostearic acid inhibits breast cancer proliferation by means of an oxidation-dependent mechanism. Cancer Prevent Res. 2009;2(10):879-86.

71. Lee-Huang $\mathrm{S}$ et al. Inhibition of MDA-MB-231 human breast tumor xenografts and HER2 expression by anti-tumor agents GAP31 and MAP30. Anticancer Res. 1999;20(2A):653-9.

72. Li C-J et al. Momordica charantia extract induces apoptosis in human cancer cells through caspase-and mitochondria-dependent pathways. Evidence-Based Complement Alternat Med. 2012;2012.

73. Li S, Tse IM, Li ET. Maternal green tea extract supplementation to rats fed a high-fat diet ameliorates insulin resistance in adult male offspring. J Nutr Biochem. 2012;23(12):1655-60.

74. Kupradinun $P$ et al. Anticlastogenic and anticarcinogenic potential of Thai bitter: gourd fruits. Asian Pacific J Cancer Prevent. 2011;12(5):1299-305.

75. Fan J-M et al. Effects of recombinant MAP30 on cell proliferation and apoptosis of human colorectal carcinoma LoVo cells. Mol Biotechnol. 2008;39(1):79-86.

76. Yasui $\mathrm{Y}$ et al. Bitter gourd seed fatty acid rich in $9 c 11 t 13 t$-conjugated linolenic acid induces apoptosis and up-regulates the GADD45, p53 and PPAR $\gamma$ in human colon cancer Caco-2 cells. Prostaglandins Leukot Essent Fat Acids. 2005;73(2):113-9.

77. Konishi $\mathrm{T}$ et al. A bitter melon extract inhibits the P-glycoprotein activity in intestinal Caco-2 cells: monoglyceride as an active compound. Biofactors. 2004;22(1):71-4.

78. Konishi T et al. Inhibitory effect of a bitter melon extract on the Pglycoprotein activity in intestinal Caco-2 cells. Br J Pharmacol. 2004;143(3):379-87.

79. Deep $\mathrm{G}$ et al. Cancer preventive potential of Momordica charantia L. against benzo (a) pyrene induced forestomach tumourigenesis in murine model system. Indian J Exp Biol. 2004;42(3):319-22.

80. Kohno $\mathrm{H}$ et al. Dietary seed oil rich in conjugated linolenic acid from bitter melon inhibits azoxymethane-induced rat colon carcinogenesis through elevation of colonic PPAR $\gamma$ expression and alteration of lipid composition. Int J Cancer. 2004;110(6): 896-901.

81. Kohno $\mathrm{H}$ et al. Dietary conjugated linolenic acid inhibits azoxymethane-induced colonic aberrant crypt foci in rats. Cancer Sci. 2002;93(2):133-42.

82. Kaur M et al. Bitter melon juice activates cellular energy sensor AMP-activated protein kinase causing apoptotic death of human pancreatic carcinoma cells. Carcinogenesis, 2013; bgt081.

83. Fang EF et al. In vitro and in vivo anticarcinogenic effects of RNase $\mathrm{MC} 2$, a ribonuclease isolated from dietary bitter gourd, toward human liver cancer cells. Int J Biochem Cell Biol. 2012;44(8):1351-1360.

84. Fang EF et al. The MAP30 protein from bitter gourd (Momordica charantia) seeds promotes apoptosis in liver cancer cells in vitro and in vivo. Cancer Lett. 2012;324(1):66-74.

85. $\mathrm{Ru} \mathrm{P}$ et al. Bitter melon extract impairs prostate cancer cell-cycle progression and delays prostatic intraepithelial neoplasia in TRAMP model. Cancer Prevent Res. 2011;4(12):2122-30.
86. Pitchakarn $\mathrm{P}$ et al. Induction of $\mathrm{G} 1$ arrest and apoptosis in androgen-dependent human prostate cancer by Kuguacin J, a triterpenoid from Momordica charantia leaf. Cancer Lett. 2011;306(2):142-50.

87. Pitchakarn $\mathrm{P}$ et al. Momordica charantia leaf extract suppresses rat prostate cancer progression in vitro and in vivo. Cancer Sci. 2010;101(10):2234-40.

88. Xiong SD et al. Ribosome-inactivating proteins isolated from dietary bitter melon induce apoptosis and inhibit histone deacetylase-1 selectively in premalignant and malignant prostate cancer cells. Int J Cancer. 2009;125(4):774-82.

89. Agrawal R, Beohar T. Chemopreventive and anticarcinogenic effects of Momordica charantia extract. Asian Pacific J Cancer Prevent. 2010;11(2):371-5.

90. Ganguly C, De S, Das S. Prevention of carcinogen-induced mouse skin papilloma by whole fruit aqueous extract of Momordica charantia. Eur J Cancer Prev. 2000;9(4):283-8.

91. Singh A, Singh SP, Bamezai R. Momordica charantia (Bitter Gourd) peel, pulp, seed and whole fruit extract inhibits mouse skin papillomagenesis. Toxicol Lett. 1998;94(1):37-46.

92. Limtrakul $\mathrm{P}$ et al. Kuguacin $\mathrm{J}$ isolated from Momordica charantia leaves inhibits P-glycoprotein (ABCB1)-mediated multidrug resistance. 2012

93. Limtrakul P, Khantamat O, Pintha K. Inhibition of P-glycoprotein activity and reversal of cancer multidrug resistance by Momordica charantia extract. Cancer Chemother Pharmacol. 2004;54(6):52530 .

94. Kai $\mathrm{H}$ et al. Inhibition of proliferation by agricultural plant extracts in seven human adult T-cell leukaemia (ATL)-related cell lines. J Nat Med. 2011;65(3-4):651-5.

95. Kobori $\mathrm{M}$ et al. Alpha-eleostearic acid and its dihydroxy derivative are major apoptosis-inducing components of bitter gourd. J Agric Food Chem. 2008;56(22):10515-20.

96. Takemoto D et al. Guanylate cyclase activity in human leukemic and normal lymphocytes. enzyme inhibition and cytotoxicity of plant extracts. Enzyme. 1981;27(3):179-88.

97. Porro $\mathrm{G}$ et al. In vitro and in vivo properties of an anti-CD5momordin immunotoxin on normal and neoplastic $\mathrm{T}$ lymphocytes. Cancer Immunol Immunother. 1993;36(5):346-50.

98. Brennan VC, Wang C-M, Yang W-H. Bitter melon (Momordica charantia) extract suppresses adrenocortical cancer cell proliferation through modulation of the apoptotic pathway, steroidogenesis, and insulin-like growth factor type 1 receptor/RAC- $\alpha$ serine/ threonine-protein kinase signaling. J Med Food. 2012;15(4):32534.

99. Claflin AJ et al. Inhibition of growth and guanylate cyclase activity of an undifferentiated prostate adenocarcinoma by an extract of the balsam pear (Momordica charantia abbreviata). Proc Natl Acad Sci. 1978;75(2):989-93.

100. Hsu H-Y et al. Antimigratory effects of the methanol extract from Momordica charantia on human lung adenocarcinoma CL1 cells. Evidence-Based Complement Alternat Med. 2012;2012.

101. Pan WL et al. Preferential cytotoxicity of the type I ribosome inactivating protein alpha-momorcharin on human nasopharyngeal carcinoma cells under normoxia and hypoxia. Biochem Pharmacol. 2014;89(3):329-39.

102. Rajamoorthi A et al. Bitter melon reduces head and neck squamous cell carcinoma growth by targeting c-met signaling. PLoS One. 2013;8(10):e78006. 